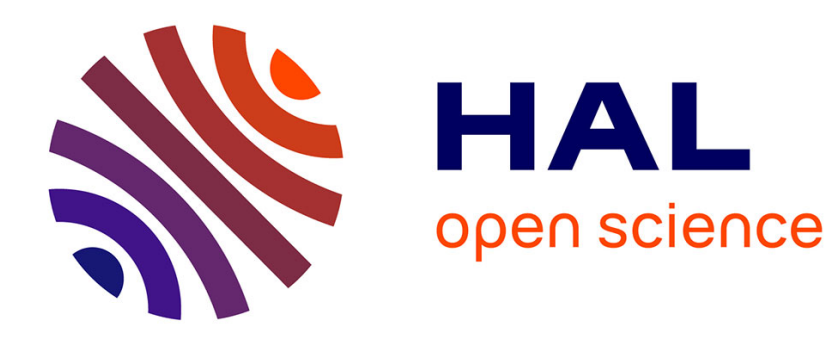

\title{
Sur une loi simple relative à la double réfraction circulaire naturelle ou magnétique
}

\author{
A. Cornu
}

\section{To cite this version:}

A. Cornu. Sur une loi simple relative à la double réfraction circulaire naturelle ou magnétique. J. Phys. Theor. Appl., 1882, 1 (1), pp.157-161. 10.1051/jphystap:018820010015700 . jpa-00237902

\section{HAL Id: jpa-00237902 https://hal.science/jpa-00237902}

Submitted on 1 Jan 1882

HAL is a multi-disciplinary open access archive for the deposit and dissemination of scientific research documents, whether they are published or not. The documents may come from teaching and research institutions in France or abroad, or from public or private research centers.
L'archive ouverte pluridisciplinaire HAL, est destinée au dépôt et à la diffusion de documents scientifiques de niveau recherche, publiés ou non, émanant des établissements d'enseignement et de recherche français ou étrangers, des laboratoires publics ou privés. 


\section{SUR UNE LOI SIMPLE RELATIVE A LA DOUBLE RÉFRACTION CIRCULAIRE NATORELLE OU MAGNÉTIQUE ( $\left.{ }^{1}\right)$;}

Par M. A. CORNU.

La propriété singulière que possèdent certains corps de faire tourner le plan de polarisation de la lumière, propriété qui paraissait ne devoir être expliquée que par une connaissance approfondie de la constitution moléculaire de ces corps, a été, par un trait de génie de Fresnel, ramenée, comme explication, aux phénomènes ordinaires de propagation des ondes.

L'explication de Fresnel est fondée en effet :

I $^{\circ}$ Sur une équivalence cinématique: une onde à vibration rectiligne équivaut à la superposition de deux ondes à vibrations circulaires de sens inverses se propageant avec la même vitesse;

$2^{\circ}$ Sur une propriété physique que Fresnel a découverte et vérifiée par expérience : dans les corps doués de pouvoir rotatoire comme le quartz, la vitesse de propagation des ondes à vibrations circulaires a deux valeurs différentes, suivant le sens de la description de la vibration : la vitesse la plus grande correspondant à la vibration circulaire de même nom que la rotation du quartz.

La théorie de Fresnel a été étendue immédiatement à l'explication du pouvoir rotatoire que le magnétisme développe dans les milieux transparents; l'adaptation était particulièrement naturelle après les beaux travaux d'Ampère sur l'identité des aimants et des solénoïdes. Cette extension a paru si naturelle, que la vérification expérimentale de la théorie de Fresnel appliquée aux phénomènes magnétiques n'a été, sinon faite, du moins publiée que dans ces derniers temps $\left({ }^{2}\right)$.

Les vitesses $v^{\prime}, v^{\prime \prime}$ des deux ondes circulaires inverses sont liées à l'arc $\alpha$ de rotation du plan de polarisation par la relation qu'on

(') Comptes rendus des séances de l'Académie des Sciences, t. XCII.

$\left({ }^{2}\right)$ A. Righ, Nuovo Cimento, t. IV, 1878 ; H. BecQuerel, Comptes rendus, t. LXXXVIII, p. 33 4.

J. de Phys., $2^{\mathrm{e}}$ série, t. I. (Avril 1882.)

II 
déduit de la théorie de Fresnel,

$$
\alpha=\frac{\pi e}{\lambda}\left(\frac{V}{v^{\prime}}-\frac{V}{v^{\prime \prime}}\right),
$$

où $\lambda$ est une longueur d'onde dans l'air de la radiation simple employée, V la vitesse de la lumière dans l'air, e l'épaisseur du milieu et $\pi$ l'arc d'une demi-circonférence, égal à 3, I 4 I 59.

J'ai été amené à rechercher une seconde relation entre ces vitesses, de manière à les déterminer toutes deux d'une manière complète; les faits observés peuvent se résumer sous la forme très simple que voici :

$\mathbf{1}^{\circ}$ Dans le quartz, la moyenne des vitesses de propagation suivant l'axe optique des ondes circulaires de sens inverses est sensiblement égale à la vitesse de l'onde ordinaire perpendiculairement à cet axe.

$2^{\circ}$ Dans le flint lourd de Faraday, la moyenne des vitesses de propagation des ondes circulaires de sens inverses séparées par l'action magnétique est sensiblement égale à la vitesse commune de ces ondes quand l'action magnétique est nulle.

Expériences faites sur le quartz. - La propriété énoncée a étè vérifiée sur toute l'étendue des radiations comprises entre le rouge et la limite des radiations ultra-violettes, c'est-à-dire depuis la raie $n^{\circ} 1$ du cadmium $(\lambda=643,7)$ jusqu'aux raies $n^{\circ} 32$ de l'aluminium $(\lambda=185)$. La méthode du prisme permet, en effet, de mesurer simultanément les trois vitesses en question : il suffit d'employer, fixés l'un au-dessus de l'autre, deux prismes de quartz ayant exactement leurs faces dans le mème plan, l'un taillé suivant la coupe précédemment indiquée, le plan bissecteur de l'angle réfringent normal à l'axe optique, l'autre ayant l'arête parallèle à cet axe. Ce double prisme, placé sur la plate-forme du goniomètre de Babinet, donne quatre images de la fente éclairée avec une lumière monochromatique; trois d'entre elles sont très voisines et parfaitement équidistantes, si les deux prismes ont exactement le même angle ( $\left.{ }^{1}\right)$. Celle du milieu est polarisée rectilignement,

(1) Il s'est présenté une particularité singulière qui m'a arrèté pendant quelque temps. Pour obtenir l'égalité parfaite des angles, j'avais fait tailler ensemble les 
parallèlement à l'arête commune : c'est l'onde ordinaire. Les deux autres sont polarisées circulairement en sens inverses, comme dans l'expérience citée précédemment. La quatrième image représente l'onde extraordinaire.

L'observation micrométrique directe ne comporte qu'une approximation médiocre dans la région des radiations visibles, en raison de la faible distance angulaire des images; mais, dans la région ultra-violette, les mesures des clichés photographiques acquièrent une précision qui croît très vite avec la réfrangibilité, car la distance des images réfractées croît comme le pouvoir rotatoire, c'est-à-dire plus vite que l'inverse du carré de la longueur d'onde. De plus, l'influence relative d'une petite inégalité dans l'angle des deux prismes décroît rapidement avec la réfrangibilité : il en résulte que la netteté des vérifications est d'autant plus grande que la longueur d'onde est plus courte, contrairement à ce qui a lieu pour les lois seulement approximatives, comme la loi de Biot, où les divergences s'aggravent avec la réfrangibilité des radiations observées.

L'insuffisance de précision que donne la méthode du double prisme avec les radiations visibles m'a conduit à compléter les

deux blocs de quartz; malgré cette précaution les angles des deux prismes n'étaient pas parfaitement égaux. Je fis recommencer le travail des surfaces : la même différence et dans le mème sens se présenta encore; je fis alors construire séparément deux nouveaux prismes par deux habiles opticiens, en recommandant le plus grand soin dans la taille des faces : la même anomalie se présenta avec les deux doubles prismes comme précédemment. J'en conclus que l'anomalie était inhérente à la nature même du quartz: je ne tardai pas à reconnaître que l'inégalité de la dilatation du quartz dans les diverses directions en était la cause. Un calcul très simple, fondé sur les coefficients donnés par M. Fizeau, me permit en effet de déterminer la température à laquelle l'angle réfringent variable devient égal à l'angle réfringent fixe et à reconnaître que cette température, voisine de $30^{\circ}$, était celle que devait prendre le quartz pendant le travail de la taille et du polissage. Il suffit en effet de tenir le prisme dans les mains pendant quelques minutes pour constater, par l'observation des images réfractées, que les deux angles réfringents deviennent égavix.

On annule l'erreur provenant de cette cause en fixant des lames de glace parallèles à l'aide d'une goutte d'un mélange d'essences de girofle et de canelle ayant le mème indice que le rayon ordinaire. Pour l'observation des radiations ultraviolettes les lames de verre étaient remplacées par des lames de quartz et le mélange d'essences par la glycérine: l'indice est un peu plus faible qu'il ne faut pour obtenir la compensation exacte, mais l'observation fournit le moyen de calculer la correction. 
mesures par la méthode des interférences. L'expérience consiste à faire interférer deux faisceaux traversant respectivement deux blocs parfaitement égaux de quartz ('), l'un dans le sens de l'axe optique, l'autre dans le sens perpendiculaire (biquartz à axes croisés). En polarisant la lumière blanche employée pour éclairer la fente lumineuse, de manière à ne laisser passer dans le second bloc que l'onde ordinaire, on observe deux systèmes latéraux de franges polarisées circulairement en sens inverses. A l'aide d'un compensateur spécial (qui n'est autre qu'un double tube d'Arago), on amène successivement la frange similaire de chaque système sous le réticule, et la moyenne des déplacements donne exactement la position de la frange centrale du système qu'on obtient en faisant passer les deux faisceaux simultanément à travers le même bloc. L'apparition de ce nouveau système de franges s'obtient par une légère translation transversale donnée au biquartz $\left({ }^{2}\right)$.

Expériences faites sur le flint lourd. - La loi relative au pouvoir rotatoire magnétique, en raison de la faible double réfraction développée et de l'opacité du flint lourd pour les radiations réfrangibles, n'a pu être établie que par la méthode des interférences. Les deux faisceaux passaient respectivement à travers deux blocs égaux de flint, l'un placé entre les deux armatures du gros électro-aimant de l'École Polytechnique, l'autre soustrait à l'action magnétique, soit par un éloignement suffisant, soit par l'insertion dans l'intérieur de l'une des armatures. La fente lumineuse était éclairée avec de la lumière polarisée circulairement vers la gauche dans la moitié supérieure, vers la droite dans la moitié inférieure $\left({ }^{3}\right)$. On obtient ainsi deux systèmes de franges, exactement sur le prolongement l'un de l'autre, lorsque le courant est inter-

(') On rencontre une anomalie analogue à celle qui a été signalée ci-dessus avec, les prismes : on l'élimine de la même manière, en fixant sur chaque extrémité du double bloc une lame de crown bien parallèle avec une goutte d'un mélange d'essences ayant l'indice ordinaire du quartz.

( ${ }^{2}$ ) Je tiens à remercier M. J. Duboscq et M. Léon Laurent pour le concours empressé qu'ils m’ont apporté dans ces expériences et l'habileté qu'ils ont déployée dans la taille et le polissage des appareils de quartz dont j'ai eu besoin.

$\left({ }^{3}\right)$ A l'aide d'un prisme de Nicol et de deux lames mica quart d'onde. A cet effet, on coupe en deux la lame de mica à bords nets et bien rectangulaires et on colle les deux morceaux sur la fente, bord à bord, après avoir tourné l'un d'eux d'un angle droit. 
rompu; mais les deux systèmes se séparent d'une quantité proportionnelle à l'intensité du champ magnétique lorsque le courant est fermé, et la moyenne de leurs positions reproduit leur position commune primitive. L'inversion du courant échange les déviations et double la précision des mesures. Malgré la petitesse des déviations, qui atteignent à peine $\pm \frac{1}{10}$ de frange, le caractère différentiel des mesures et la précision des pointés, qui dépasse parfois $\frac{1}{200}$ de frange, permettent d'affirmer l'égalité des variations de vitesse à moins de $\frac{1}{20}$ de leur valeur, approximation qui paraîtra déjà considérable, eu égard à l'ordre de grandeur du phénomène.

Énoncé plus général. - Bien que les expériences n'aient porté que sur deux substances particulières, comme ces deux substances réunissent, chacune dans leur genre, les conditions les plus favorables à la précision des mesures, je suis convaincu que les résultats obtenus doivent s'étendre à tous les corps similaires sur lesquels il serait plus difficile d'expérimenter.

L'analogie des deux lois obtenues dans des conditions si différentes semblerait même révéler une propriété optique générale de la matière pondérable relativement à la transformation des ondes lumineuses : en effet, ces deux lois sont susceptibles d'un énoncé cominun indépendant des circonstances dans lesquelles elles ont été obtenues:

Le dédoublement d'une onde polarisée rectilignement en deux ondes polarisées curculairement en sens inverses s'effectue de manière que la moyenne des vitesses de propagation des ondes dédoublées soit égale à la vitesse de propagation de l'onde unique qui existe dans les conditions où les causes de ce dédoublement n'agissent pas.

La généralité de cette conclusion est trop éloignée des faits observés pour qu'on puisse l'accepter autrement que comme ure conjecture; aussi ne me serais-je pas hasardé à l'énoncer si je n'étais en possession de faits analogues, rendant fort probable l'existence de relations de cette forme, que j'exposerai ultérieurement. 